\title{
Improved net rewriting system-based approach to model reconfiguration of reconfigurable manufacturing systems
}

\author{
Jun Li $\cdot$ Xianzhong Dai $\cdot$ Zhengda Meng
}

Published online: 13 March 2008

(C) Springer-Verlag London Limited 2008

\section{Erratum to: Int J Adv Manuf Technol DOI 10.1007/s00170-007-1037-5}

Unfortunately, some errors occurred in the text. The corrected version is given below:

In section 2:

$C M G \quad$ Closed marked graph.

$S C M G \quad$ Sequential closed marked graph.

$C M G(k) \quad k$ - parallel closed marked graph.

$S C M G(k) \quad k$ - parallel sequential closed marked graph.

$C M G(k, l) \quad$ Variant closed marked graph with $k$ inputs and $l$ outputs.

In the first sentence of Definition 3

Definition 3 Given a marked Petri net $G=\left(P, T, F, M_{0}\right)$, where $P=P^{r} \cup P^{o}, P^{r}=\{\widetilde{p}\}$, and $\left|P^{o}\right| \geq 1, G$ is called a closed marked graph $(C M G)$, if all following conditions are satisfied:

In the first sentence of Definition 4

Definition 4 Given a marked Petri net $G=\left(P, T, F, M_{0}\right)$, where $P=P^{r} \cup P^{o},\left|P^{o}\right| \geq 1$, and $|T|>1, G$ is called a sequential closed marked graph $(S C M G)$, if

In Definition 5 statement 1, 3 and 4

1) If it is an $O M G$, then $G$ is called a $k$-parallel open marked graph $(O M G(k))$. Suppose the input and output node pair

The online version of the original article can be found at http://dx.doi. org/10.1007/s00170-007-1037-5.

J. Li $(\bowtie) \cdot$ X. Dai $\cdot$ Z. Meng

School of Automation, Southeast University,

Nanjing 210096, China

e-mail: j.li@seu.edu.cn of $G^{i}$ as $\left(t_{i n}^{i}, t_{\text {out }}^{i}\right)$, then the input node set and the output node set of $G$ are $T_{i n}=\left\{t_{i n}^{1}, t_{i n}^{2}, \cdots, t_{i n}^{k}\right\}$ and $T_{\text {out }}=$ $\left\{t_{\text {out }}^{1}, t_{\text {out }}^{2}, \cdots, t_{\text {out }}^{k}\right\}$, respectively;

$3)$ If it is a $C M G$, then $G$ is called a $k$-parallel closed marked graph $(C M G(k))$. Suppose the input and output node pair of $G^{i}$ be $\left(t_{i n}^{i}, t_{\text {out }}^{i}\right)$, then the input node set and the output node set of $G$ are $T_{i n}=\left\{t_{i n}^{1}, t_{i n}^{2}, \cdots, t_{i n}^{k}\right\}$ and $T_{\text {out }}=\left\{t_{\text {out }}^{1}, t_{\text {out }}^{2}, \cdots, t_{\text {out }}^{k}\right\}$, respectively; or

4) If it is a $S C M G$, then $G$ is called a $k$-parallel sequential marked graph $(S C M G(k))$. Suppose the input and output node pair of $G^{i}$ be $\left(t_{\text {in }}^{i}, t_{\text {out }}^{i}\right)$, then the input node set and the output node set of $G$ are $T_{\text {in }}=\left\{t_{\text {in }}^{1}, t_{\text {in }}^{2}, \cdots, t_{\text {in }}^{k}\right\}$ and $T_{\text {out }}=\left\{t_{\text {out }}^{1}, t_{\text {out }}^{2}, \cdots, t_{\text {out }}^{k}\right\}$, respectively.

In Definition 6 statement 1 and 2

1) If $P^{r}=\varnothing$ and there exists an $O M G$ type full subnet $G^{\prime}=$ $\left(P^{o}, T^{\prime}, F^{\prime}, M_{0}\right)$ in $G$, where $T^{\prime}=\left\{t_{\text {in }}^{i}\right\} \cup T_{2} \cup\left\{t_{\text {out }}^{j}\right\}$ and $F^{\prime}=F-\bigcup_{\forall t \in T_{1}, t \neq t_{i n}^{i}} F(t, \dot{p})-\bigcup_{\forall t \in T_{3}, t \neq t_{\text {out }}^{i}} F(\ddot{p}, t)$, then $G$ is called a variant open marked graph with $k$ inputs and loutputs denoted as $O M G(k, l)$;

2) If $P^{r}=\{\widetilde{p}\}$ and there exists an $O M G$ type full subnet $G^{\prime}=\left(P, T^{\prime}, F^{\prime}, M_{0}^{\prime}\right)$ of $G$ is a $C M G$, where $T^{\prime}=\left\{t_{\text {in }}^{i}\right\} \cup T_{2} \cup\left\{t_{\text {out }}^{j}\right\}, F^{\prime}=F-\bigcup_{\forall t \in T_{1}, t \neq t_{\text {in }}^{i}} F(t, \dot{p})-$ $\bigcup_{\forall t \in \tilde{p}^{*}, t \neq t_{i n}^{i}} F(\widetilde{p}, t)-\bigcup_{\forall t \in \tilde{p}^{-}, t \neq t_{\text {out }}^{i}} F(t, \widetilde{p})$, and $M_{0}^{\prime}(p)=$ $M_{0}(p), \forall p \in P$, then $G$ is called a variant closed marked graph with $k$ inputs and $l$ outputs denoted as $C M G(k, l)$.

In subsection 4.4 .1 sentence 5

This is the case that each of the machines sequentially holding its own resource turns into all of them sharing a common resource. 